\title{
Resource Allocation Optimization for Heterogeneous Network
}

\author{
${ }^{1}$ B.Manjula, ${ }^{2}$ V.Venkata Rao, ${ }^{3}$ P.Chandrasekhar Reddy \\ ${ }^{I}$ Associate Professor, Dept. of ECE, KECW, Narasaraopeta, AP, India \\ ${ }^{2}$ Professor and HOD, Dept. of ECE, NEC, Narasaraopeta, AP, India \\ ${ }^{3}$ Professor-Coordination, Dept. of ECE, JNTUH, Hyderabad, TS, India
}

\begin{abstract}
The improvement of communication throughput using a cross layer mode of communication is proposed. Due to critical mode of applications of sensor networks, it is required to achieve data at faster rate for high refreshment. As the communicating data's are sensed or measured data, it is required to have higherthroughput and less interference, to achieve accuracy and optimal resource utilization. In this paper we propose aintegrated cross layer mechanism to achieve higher performance in sensor networks. A new approach of cross layer controlling, based on integrated factor of power allocationand memory blockage is proposed.This mode of communication results at faster data transfer, with lower energy resource consumption over a wirelesssensor network architecture.
\end{abstract}

Keyword: Cross Layer communication, integrated control, sensor network, memory blockage, transmission power.

\section{Introduction}

In the process of communication, data are transferred from source to sink via different intermediate switch nodes, to perform an operation. In the industrial domain these data are various sensed data, which are captured from different measuring units and transferred to a centralized monitoring point. In this process data is captured and transferred via a communication medium. This medium is a fixed wire line cable or a wireless channel through which data is transferred. During the process of transmission it is required to transmit the data at faster rate and should be as accurate as possible. In concern to accuracy wired lines are very optimal as, in this mode of communication; data are travelled over a dedicated closed medium. However the under laying cost and the maintenance of such network is very complicated. To achieve economical mode of communication, wireless communication is more preferred. However in this approach accuracy of data is more concern. As well in such approach a dedicated interlink point, termed as base station (BS) is required to switch the data. In various real time application installation of BS and transferring data over it get constraint, such as oceanic application, mining, large distributed industrial units etc. For such usage wireless sensor network is a promising solution. In the application of sensor network, the sensors are installed at the measuring terminals and the acquired data are encoded, forwarded via intermediate links. During the process of transmission, these measured data requires more accurate transmission at faster rate. To achieve the objective in this paper we propose an enhancement approach to achieve faster data delivery ratio for wireless sensor network. With the objective of obtaining higher throughput in wireless sensor network, various approaches were made in past. With controlling of interference logic to improve throughput in [3], a pruning algorithms was presented, where the transmission power is controlled to interference at each node. A linear programming model is presented for routing to maximize the throughput factor. In other approaches, Joint routing and rate allocation approach were proposed to improve performance in [4]-[5]. A joint power controlling and scheduling approach is defined at physical layer and network layer in [6]. In [7] to control interference in the links, two simultaneous requests were not processed for a common node at the same time slot. Towards performance improvement, in [2] a congestion control mechanism is presented, which controls the flow of data based on the memory overhead conditions. The Congestion Control and Fairness (CCF) routing scheme [8] Reads the packet processing time as a monitoring factor for congestion. However the processing time may not be appropriate in congestion monitoring as the rate of transmission may vary over time. In the Priority-based Congestion Control Protocol (PCCP) [9] the packet service and time factor is used as a monitoring parameter. However in such method, the packet buffering conditions are not considered.The CODA protocol [10] uses hop-by-hop and end-to-end congestion control scheme to control the congestion by discarding packets at the node.Similar to CODA, in Fusion [11] a static threshold based congestion controlling is proposed. However this method is very limited under dynamic channel conditions. The interference-aware fair rate control (IFRC) protocol [12] also uses a static threshold approach, where the link rate is controlled via the threshold operation. With all these approaches it was focused to improveperformance either via power controlling or via congestion controlling mechanism. Where power controlling is performed at physical layer and scheduling of packet transmission is performed at MAC layer. However with high demand in processing precision in sensor network, a single layer controlling is not 
sufficient. A cross layer controlling with the integration of different controlling parameters is required. With this objective in this paper, a cross layer control mechanism focusing on throughput enhancement based on power allocation and congestion controlling is proposed. In recent past an optimal power allocation scheme based on interference controlling is developed in [1]. This approach presented a maximum power allocation problem into two simpler linear programs for symmetric and asymmetric links. In [2] with the similar objective to improve performance, a congestion avoidance approach is proposed. However no approach was observed controlling these two factors, to achieve higher performance. With this approach in this paper a new integration approach "Joint-CL" for performance enhancement in sensor network is proposed, under two different layers controlling.

The rest of the paper is outlined into 6 sections. The approach of interference based power allocation to improve throughput is outlined in section 2. In section 3 the approach of cross layer coding for congestion controlling is proposed. The proposed integrated model of throughput enhancement is outlined in section 4 . The experimental results for the developed system over the conventional approaches were outlined in section 5. The paper is concluded in section 6 .

\section{Interference Controlled Power Allocation [1]}

For the throughput improvement in wireless sensor network, a power control mechanism was proposed in [1]. The Transmission power was controlled to achieve the objective of higher throughput, controlling the factor of interference for the wireless sensor network. For achieving the objective of higher throughput, a linear programing model for minimal total power allocation is developed. In the method of power based interference management, the minimal total power allocation is defined by a minimum power allocation algorithm. For a symmetric link scenario the a link status $C_{i, j}$ is used, which is set as ' 1 ' for available link and ' 0 ' for no link condition. In the case of asymmetric link acknowledgment is not considered, in such as case $\mathrm{C}_{\mathrm{i}, \mathrm{j}} \neq \mathrm{C}_{\mathrm{j}, \mathrm{i}}$. Hence the power allocation $P_{i, j}$ for a data transfer from $i->j$ will not be same as $P_{j, i}$. The allocable power is then subjected to maximization of source data rate $\mathrm{R}_{\mathrm{i}}$.

Subjected to,

$$
\max \left(\sum_{i \in \text { sources }} R_{i}\right)
$$

$$
\sum_{j \in N_{i}} R_{i j}-\sum_{j \in N_{i+}} R_{j i}=R_{i}
$$

Where $\mathrm{N}_{\mathrm{i}}$ is the number of nodes that source ' $\mathrm{i}$ ' can reach and $\mathrm{N}_{\mathrm{i}}{ }^{+}$is the number of nodes that can reach ' $\mathrm{i}$ 't node.

In the case of symmetric link condition, the nodes are communicated with an acknowledgment. In such case, the network nodes are two way, and in such case, $\mathrm{C}_{\mathrm{ij}}=\mathrm{C}_{\mathrm{ji}}$. In such case the interference level is defined as,

Subjected to,

$$
\max \left(\sum_{i \in \text { sources }} R_{i}\right)
$$

$$
\sum_{j \in N_{i}}\left(R_{i j}-R_{j i}\right)=R_{i}, \forall i
$$

A topology selection is carried out for the links satisfying the required criterion. A transportation of data over the selected topology will result in higher throughput, due to the constraint criterions of allocable power level. However this approach develops and communication system, considering linear traffic model. The traffic model and node characteristic is not considered. However when the traffic is non-linear, there will be huge traffic flow from each node. Hence, power interference doesn't provide the maximum throughput of the system. So as to achieve higher throughput, considering node characteristic, congestion avoidance coding were developed.

\section{CL-APCC Method [2]}

Towards the development of congestion controlling at each node, a cross layer active predictive congestion control (CL-APCC) is proposed in [2]. This approach applies a network flow analysis of the memory status for each node in the network. This approach state that, For a occupied node memory size of ' $L$ ', with the maximum node memory size of ' $m$ ', with a threshold value of $L_{\max }$, the congestion control method of the nodelevel is as applied. If the occupied node memory size is $L<L_{\max }$, CL-APCC identifies the node is not congested. If the memory size is $L_{\max }<L<m$, then CL-APCC identifies the congestion of node-level to have probably occurred. In this case, the sending rate of the node is adjusted to control the congestion. If the occupied node memory size reaches the maximum $(L=m)$, the input rate of the node is adjusted to 0 . Secondly, the congestion control method of the system-level is outlined. At the beginning of a new period ' $t$ ', CL-APCC protocol makes a 
prediction for the expected value $E(\mathrm{t})$ of data quantity in period ' $t$ ' using the average rate in period ' $t-1$ ' in the network. If $E(t)<n \times L \max$, where ' $n$ ' is the number of nodes in the grid, congestion of system-level does not occur. On the contrary, if $n \times m>E(t)>n \times L$ max, congestion of system-level may occur. In this case, the average inputting and outputting rates of the network are adjusted to avoid system-level congestion. To adjust the outputting rate the probability of transportation is computed. The probability of congestion is derived as a function of the memory length, and the traffic is controlled so as avoid back pressure at the source side. The derived approach is a cross layer controlling of traffic at network and MAC layer. However the approach is constraint with the controlling of traffic based on the memory status of the node.

\section{Proposed Joint-Cl Approach}

It is observed that, in wireless sensor network, the throughput is effective by the inference offered as well the node congestion available. Hence a joint analysis of the two factors is required to have optimal controlling of traffic flow and throughput enhancement. To achieve this objective a joint cross layer controlling algorithm is proposed. The proposed approach is as outlined below.

For the allocated power of $P_{i}$, derived from the interference model from [1], at node $n_{i}$, considering the traffic blockage at, a node $\mathrm{n}_{\mathrm{i}}$ as $\mathrm{B}_{\mathrm{i}}$, the observed probability of congestion level is defined as [2],

i.e $P_{\text {con }}=\sum_{L=L \max }^{m} P_{L}$.

$$
P_{\text {con }}=P_{\text {Lmax }}+P_{L \max +1}+\cdots \ldots .+P_{m} .
$$

However, this blockage rate is purely based on the occupied memory condition. And no interference Consideration is made.To have a successful transmission, it is required to have, $P_{i} \geq X_{i j} P_{i j}$

Where, $X_{i j}$ - is the status of link and

$P_{i j}$ - is allocated power for a link between $\mathrm{i}$ and $\mathrm{j}$

However, this allocated power is to be controlled by, traffic conditions.

Hence for a network topology with blockage probability ' $\mathrm{P}_{\text {con }}$ ' the probable selection is made for the link satisfying min $\left(P_{c o n}\right)$, having $P_{i}$ power selected. However selecting of such paths would become an open loop issue as certain cases this optimal path may not-be obtained. Hence, to obtain path with interface control and congestion control, a feedback acknowledgment to the source is made. Where the feedback data consist of the memory blockage at the link node. Let ' $f_{i}$ ' be the blockage level at the link node ' $n_{i}$ ', to obtain lower congestion rate, the allocable power should be such controlled that, interference is minimized and the back pressure to source is avoided. To achieve this, a power scheduling algorithm with congestion factor considered is proposed. The proposed algorithm for the cross layer controlling is as outlined,

Algorithm:

Source: Input -' $\mathrm{f}_{\mathrm{i}}$ '- blockage level obtained from Acknowledgment

- ' $P_{i}$ ' - allocable power in the node.

Output - ' $\mathrm{P}_{\mathrm{o}}$ ' - allocated power.

For, the allocation algorithm processing each node,

While,

Sink_ID $\neq$ current_ID

Initialize,

Set, Link status, $\mathrm{C}_{\mathrm{i}}=1$

Power allocated, $\mathrm{P}_{\mathrm{i}}-1=\mathrm{P}_{\mathrm{i}}$.

Feedback, congestion $\mathrm{f}_{\mathrm{i}}-1=\mathrm{f}_{\mathrm{i}}$.

do,

Compute allocable power $\mathrm{P}_{\mathrm{i}+1}$ as,

$P_{i+1}=\left(C_{i} . P_{i}\right)$.

If, current blockage rate, $\mathrm{f}_{\mathrm{i}}<\mathrm{L}_{\max }$

$P_{\text {alloc }}=C_{i} P_{i}$

else

$$
P_{\text {alloc }}=C_{i} P_{i} * K
$$

end

end

Where, $\mathrm{L}_{\max }$ is maximum allowed memory overhead at each node. And ' $\mathrm{K}$ ' is the proposed decimating factor. The value of $\mathrm{K}$ varies from 0 to 1 . The value of $\mathrm{K}$ varies based on current blockage rate. The value ' 0 ' indicates the least power allocated and the value ' 1 ' indicates the maximum power allocated. As with the increase in blockage rate, interference increase and back pressure to the source increase, hence, if the power is 
allocated only w.r.t. unavailable interference margin the optimization is not achieved. In the proposed approach however the feedback blockage rate ' $f_{i}$ ' is then used as one decision factor to allocate the transmitting power.

The decimating factor, $\mathrm{K}$ is defined as a function of ' $\mathrm{f}\left(\mathrm{f}_{i}\right)$ ' defined by,

$$
K=\left(L_{\max }-f_{i}\right) \text {. }
$$

To achieve higher controllability, each node unit is optimized with a variant of two bound regions, of minimum and maximum blockage limits, so, as to achieve traffic flow under high congestion probability. In the case of variant traffic, the memory unit is non-linear in nature, and the link block will then vary from $\mathrm{L}_{\min }$ to $\mathrm{L}_{\max }$.

Hence, the decimation factor, $\mathrm{K}$ then be, defined as,

Where, Pa- probability of blockage.

$$
K=P_{a}\left(L_{\max }-f_{i}\right)
$$

Define by,

Where,

$$
P_{a}=\frac{P_{b}}{1-P_{c}}
$$

$\mathrm{P}_{\mathrm{c}}$ - Number of packets been transferred

$\mathrm{P}_{\mathrm{b}}$ - Blockage ratio, defined by,

$$
P_{b}=L_{\max } \times\left(\frac{f_{i}-L_{\min }}{L_{\max }-L_{\min }}\right)
$$

With the allocation of power based on these factors the congestion factor as well the interference factor are considered. The throughput of the system is hence improved, as the congestion level and the interference are controlled simultaneously.

\section{Experimental Results}

To evaluate the proposed approach a simulation model is developed considering the following network conditions,

\begin{tabular}{|l|l|}
\hline Network parameter & Values \\
\hline Node placement & Random \\
\hline Routing Protocol & AOMDV \\
\hline MAC protocol & IEEE 802.11 \\
\hline Power allocation & Random \\
\hline Transmission range & $40 \mathrm{~m}$ \\
\hline Network area & $200 \times 200$ \\
\hline Number of nodes & $50-1000$ \\
\hline Memory size / node $(\mathrm{M})$ & $3 \mathrm{M}$ \\
\hline $\mathrm{L}_{\min }$ & $0.15 \mathrm{xM}$ \\
\hline $\mathrm{L}_{\max }$ & $0.75 \mathrm{xM}$ \\
\hline Initial blockage probability & 0.1 \\
\hline
\end{tabular}

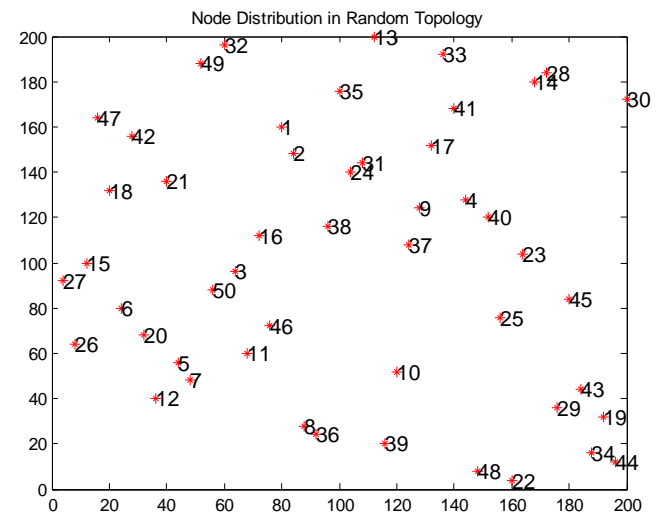

Figure 1: A randomly distributed network topology

For the simulation of the proposed approach a randomly distributed network is developed. The network is defined with 50 independent nodes, acting as routers. Each node is dynamically placed over a $200 \times 200$ network area. The allocated resources of bandwidth and power are randomly distributed for each node. The node with their geographical location and node ID is shown in figure 1. 


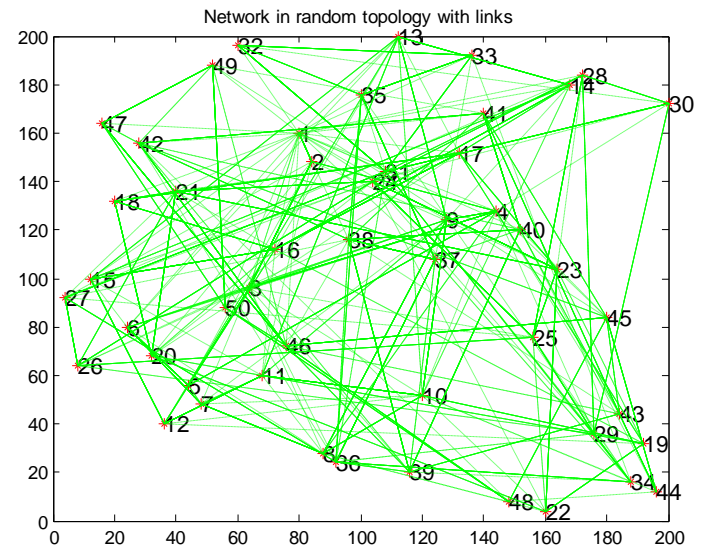

Figure 2: Possible links for each node to its 1-hop neighbor

For the generated random network, all probable links are obtained. A communication range of 40 units is defined, and a based on the Cartesians distance formula between two points, distance is computed. Nodes satisfying the range constraints are declared as 1-hop neighbors. Each node is defined with its ID as source and all other in range nodes as neighbor nodes. The obtained possible links for all the distributed node is as shown in figure 2 .

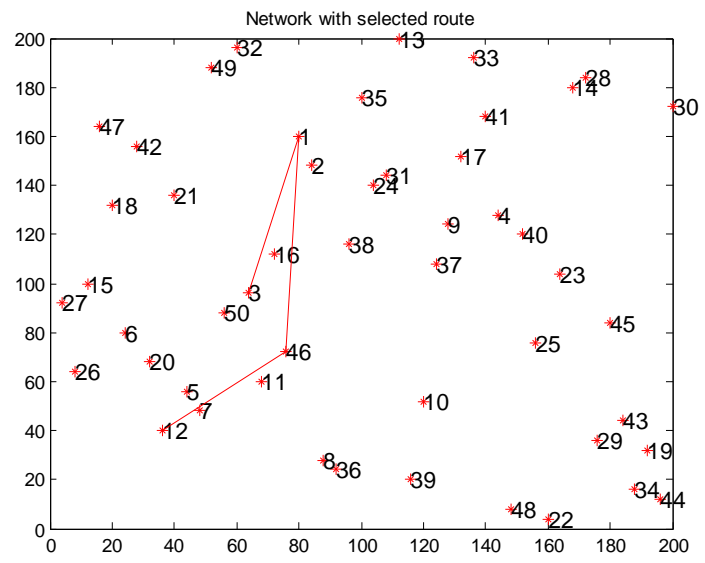

Figure 3: Selected path for data forwarding

An on demand routing algorithm called AODV routing is carried out. The algorithm performs a forward and backward tracing over all links and compute all possible path from a source to the corresponding distance. Among all obtained paths with highest offered bandwidth, highest power and shorter path is selected. One selected path considering source as 3 and destination as 12 is shown in figure 3 .

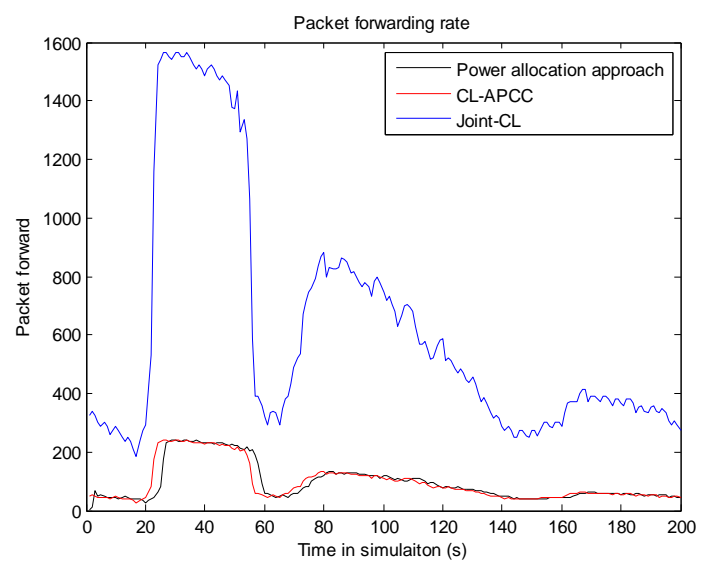

Figure 4: Number of packet forward 
Over the selected route, a packet of 600 bytes of data is transferred. For the transmission of the data, optimization approach based on power interference controlling [1], cross layer coding [2] and proposed joint cross layer coding is carried out. The packet forwarding of the three methods are then recorded. The packet forwarding over time of simulation is presented in figure 4. It is observed that, the rate of packet forwarding for joint controlling is higher than the two conventional methods. As the joint control method, allocate the transmission power and corresponding routing based on current interference and blockage observed, the forwarding of packet is higher, and hence the back pressure to the source is decreased.

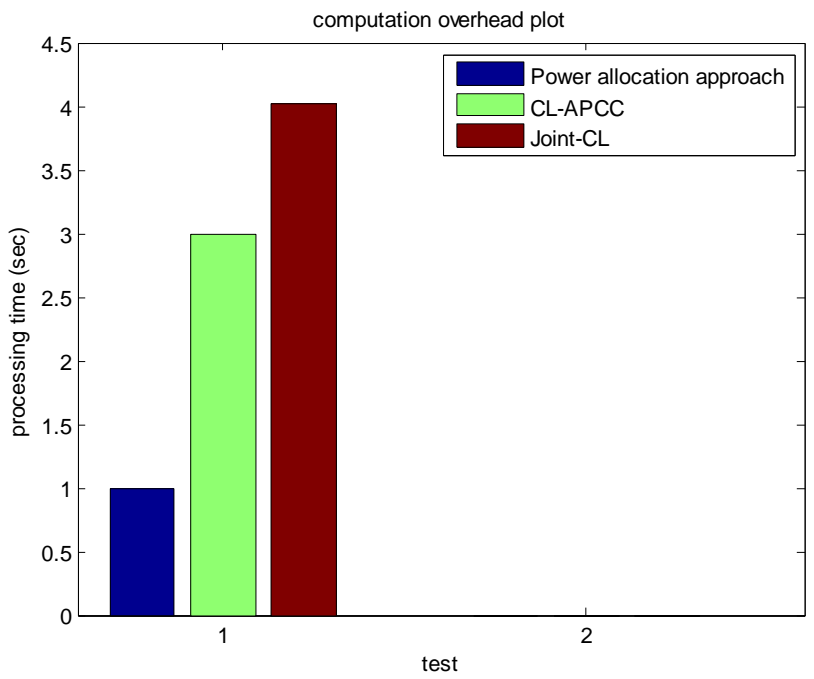

Figure 5: Overall processing time for the three methods

To perform the operation of resource scheduling, each method has its own computational time. The observed communication time for the developed three methods is as shown in figure 5. It is observed that the computation time for interference based controlling mechanism is very low than the other two method. However in the case of memory control mechanism [2], the time taken is higher due to the computation of memory buffering and optimization of allocation. However for the proposed approach, the processing time is still higher about 1.5 units, due to the optimization of multiple factors simultaneously. However this overhead is comparable for the obtained network metrics, as outlined below.

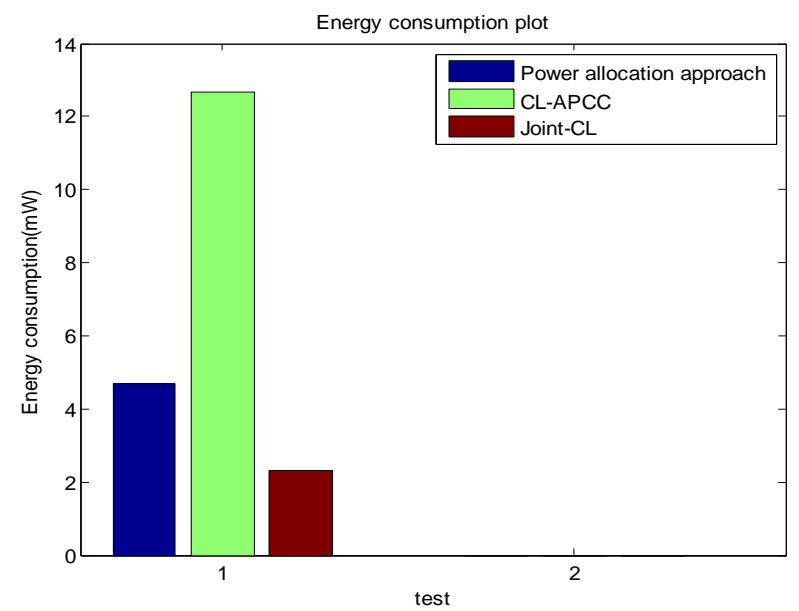

Figure 6: Overall energy consumption for the three methods

The energy consumption for the three methods are compared, and the proposed joint CL optimization, results in very low energy consumptions, in compared to the other two methods. This is achieved due to the controlling of allocation based on both factors of interface and blockage together, rather than independent controlling. 


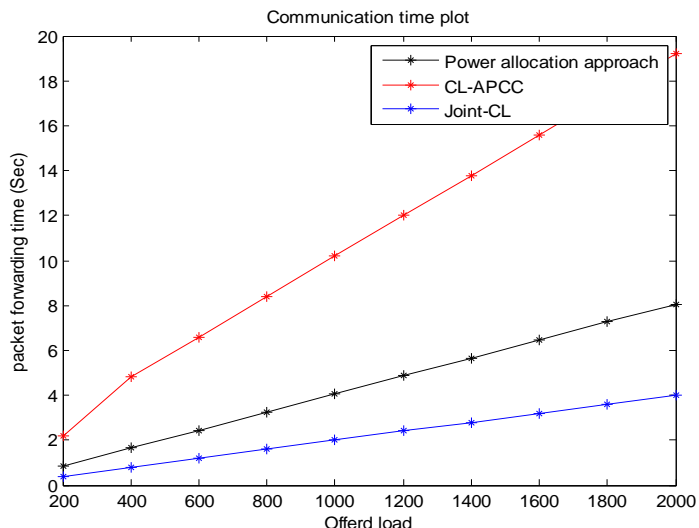

Figure 7: Time for packet forwarding with offered load

To analyze the effect of the proposed method over the other two methods, an analysis is carried out for different offered load for transmission. The simulation is carried out for a node density of 50 nodes, with a random node position and its characteristics. It is observed that, the packet forwarding time for CL-APCC method goes very high, due to the recursive computation of the memory buffer computation and allocation. However, in the proposed method with consideration of blockage factor, interference is also considered, Hence, in this approach, the forwarding time is reduced. The allocation of memory is dynamic in the proposed approach which varies between a minimum and a maximum limits. The power allocation over this variant buffer limits is done via interference consideration, resulting in faster forwarding of packets.

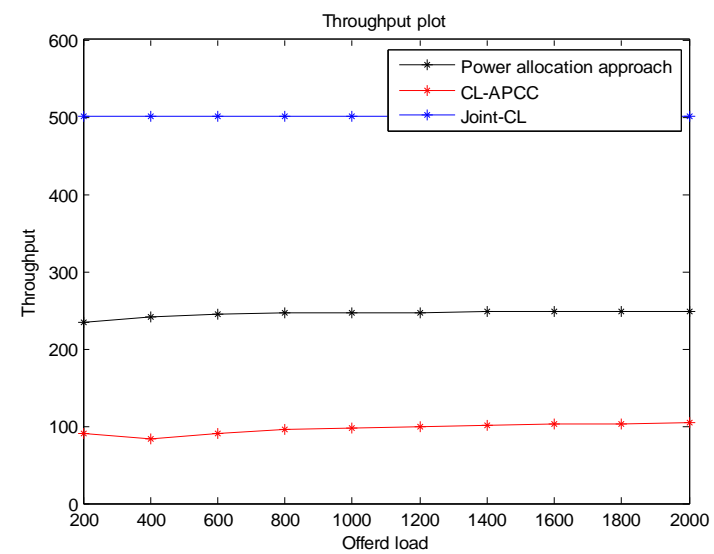

Figure 8: Throughput plot for the three methods for different offered load

As the packet transmission is faster in the proposed joint-CL method, the observed throughput is also higher. The throughput is found to be stable over a unit of offered load. With the increase in the offered load the interference and congestion both are controlled, hence retaining a high throughput for proposed method.

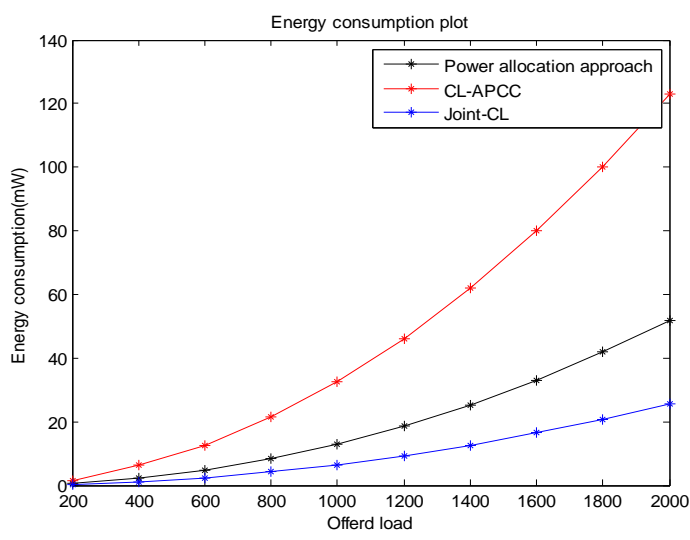

Figure 9: Total energy consumed for the three methods 
The total consumed energy for packet forwarding and reception is presented on figure 9 . It is observed that, the energy consumed for the proposed joint-CL method is lowered due to proper resource allocation, reducing resource wastage. However, in other two methods, the energy gets wasted under high congestive passage, even under control of interference and hence demands for higher energy consumption. In the similar manner in CL-APCC approach, the consumption increase if the interference id observed higher, even under low blockage paths.

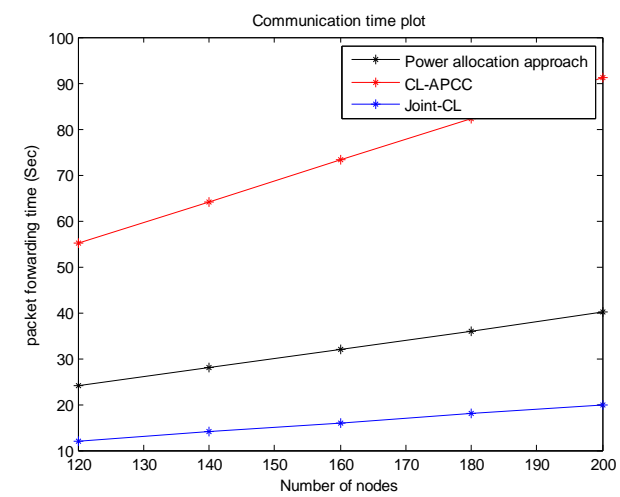

Figure 10: Packet forwarding over variation in node density

With the variation of node density, it is observed that, the time for forwarding packet is high in the case CL-APCC method. This approach get constraints due to the generation of more probable intermediate links at the path, which results in more computation in controlling and allocation. This increase the overall time for packet forwarding. Even for optimizing of blockage factor, it is observed that, the controlling is made based on the upper limit of the queue size, $\mathrm{L}_{\max }$, this results in high back pressure to the source node, which adds up to the computation effort, making forwarding slower.

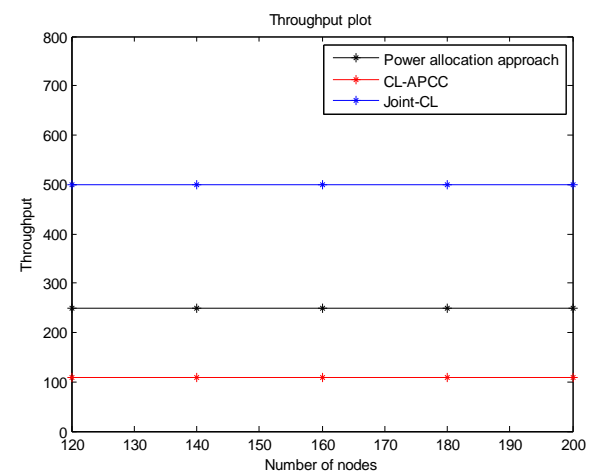

Figure 11: Throughput comparison over variation in node density

Due to higher probable links the forwarding is expected faster. However as links are more, the probability of interference and intern the blockage is also more. Hence, a joint optimization will results in faster transmission, than the individual controlling. This results in higher throughput, as observe in figure 11.

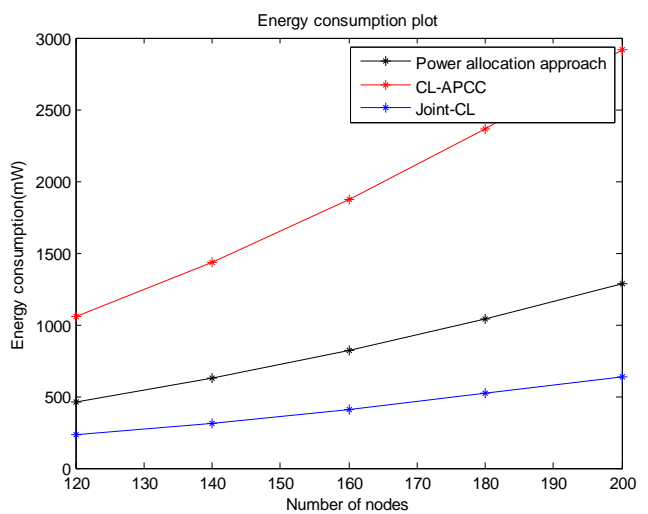

Figure 12: Energy utilization for packet forwarding over different node density 
Due to optimal resource allocation, the energy allocated for transmission, reception is also optimized. In the case of joint CL method this is observed to be very low as in comparison to the other two methods. However it is observed that the total energy consumption increase with increase in number of nodes. This is because of more intermediate nodes used for forwarding data, increasing overall energy consumption.

\section{Conclusion}

A joint controlling approach for throughput improvement is proposed. The interference controlled power allocation and memory constraint congestion controlling approaches were analyzed, and a cross layer modeling, controlling at physical layer and MAC layer is proposed. The joint control algorithm is applied over each node in the network with the consideration of both interference level and blockage factor. A power allocation is controlled with the feedback congestion factor. To achieve a higher throughput for variant traffic condition, a 2-bound controlling logic is presented. This controlling approach improves the throughput of the system, and minimizes the energy dissipation.

\section{References}

[1]. Maggie X. Cheng, Xuan Gong, Lin Cai, and XiaohuaJia, "Cross-Layer Throughput Optimization With Power Control in Sensor Networks", IEEE Transactions on Vehicular Technology, Vol. 60, No. 7, September 2011.

[2]. Jiangwen Wan, XiaofengXu ,RenjianFeng and Yinfeng Wu, "Cross-Layer Active Predictive Congestion Control Protocol for Wireless Sensor Networks", Sensors Vol.9. No.10, 2009

[3]. D. Li, B.Wang, and X. Jia, "Topology control for throughput optimization in wireless mesh networks," in Proc. 4th Int. Conf. MSN, Dec. 10-12, 2008.

[4]. Xianren Wu and ZhiTian, "Optimized Data Fusion in Bandwidth and Energy Constrained Sensor Networks", ICASSP,IEEE, 2006

[5]. M. X. Cheng, X. Gong, and L. Cai, "Joint routing and link rate allocation under bandwidth and energy constraints in sensor networks," IEEE Trans. Wireless Commun., vol. 8, no. 7, pp. 3770-3779, Jul. 2009.

[6]. K. Jain, J. Padhye, V. N. Padmanabhan, and L. Qiu, "Impact of interference on multi-hop wireless network performance," in Proc. 9th Annu. Int. Conf. MobiCom, 2003, pp. 66-80.

[7]. J. Tang, G. Xue, C. Chandler, andW. Zhang, "Link scheduling with power control for throughput enhancement in multihop wireless networks," IEEE Trans. Veh. Technol., Vol. 55, No. 3, pp. 733-742, May 2006.

[8]. Cheng TienEe, Bajcsy, R., "Congestion control and fairness for many-to one routing in sensor networks" In Proceedings of the $2^{\text {nd }}$ ACM Conference on Embedded Networked Sensor Systems, Baltimore, MD, USA, November, 2004.

[9]. Chonggang W., Sohraby. K., Lawrence. V, Li. B, Hu Y.M., "Priority-based congestion control in wireless sensor networks", IEEE International Conference on Sensor Networks, Ubiquitous, and Trustworthy Computing, Taichung, Taiwan, June, 2006.

[10]. Wan C.Y., Eisenman. S., Campbell A., "CODA: Congestion detection and avoidance in sensor networks, ACM Conference on Embedded Networked Sensor Systems, Los Angeles, CA, USA, November, 2003.

[11]. Hull.B., Jamieson.K.,Balakrishnan. H., "Mitigating congestion in wireless sensor networks", ACM conference on Embedded Networked Sensor Systems, New York, NY, USA, November, 2004.

[12]. Rangwala. S., Gummadi. R., Govindan. R., Psounis. K., "Interference aware fair rate control in wireless sensor networks ACM SIGCOMM'06, Pisa, Italy, September, 2006 